\title{
New data on the scutigeromorph and scolopendromorph centipedes (Chilopoda: Scutigeromorpha: Scutigeridae; Scolopendromorpha: Scolopendridae, Cryptopidae) from Kazakhstan
}

\section{Новые данные о мухоловках и сколопендровых многоножкках (Chilopoda: Scutigeromorpha: Scutigeridae; Scolopendromorpha: Scolopendridae, Cryptopidae) из Казахстана}

\author{
Yu.V. Dyachkov \\ Ю.В. Аьячков
}

Altai State University, Lenin Avenue, 61, Barnaul 656049, Russia. E-mail: dyachkov793@mail.ru
Алтайский государственный университет, проспект Ленина, 61, Барнаул, 656049 Россия

KEY WORDS: new faunistic records, key, Central Asia.

КЛЮЧЕВЫЕ СЛОВА: новые фаунистические находки, ключ, Центральная Азия.

ABSTRACT. The scutigeromorph fauna of Kazakhstan comprises at least three species in three genera in one family, while the scolopendromorph one consists of three species in two genera in two families. The family Cryptopidae, the genus Cryptops, and the species $C$. doriae Pocock, 1891 and C. hortensis (Donovan, 1810) are new to the Kazakhstan list, while $C$. doriae in also new to the entire Palaearctic. Scolopendra canidens Newport, 1844 is new to Kazakhstan's Kyzylorda and Turkestan regions, while Scutigera coleoptrata (Linnaeus, 1758) to the Akmola Region. Some morphological characters of scolopendromorph species are discussed and illustrated. An identification key to the scutigeromorph and scolopendromorph centipedes of Kazakhstan is given. All records are mapped.

How to cite this article: Dyachkov Yu.V. 2020. New data on the scutigeromorph and scolopendromorph centipedes (Chilopoda: Scutigeromorpha: Scutigeridae; Scolopendromorpha: Scolopendridae, Cryptopidae) from Kazakhstan // Arthropoda Selecta. Vol.29. No.2. P.173-184. doi: 10.15298/arthsel. 29.2.02

РЕЗЮМЕ. Фауна многоножек-мухоловок Казахстана составляет, как минимум, три вида из трех родов одного семейства; тогда как фауна сколопендровых многоножек представлена тремя видами из двух родов и двух семейств. Семейство Cryptopidae, род Cryptops и виды C. doriae Pocock, 1891 и $C$. hortensis (Donovan, 1810) являются новыми для фауны Казахстана: кроме того, $C$. doriae впервые отмечен в Палеарктике. Scolopendra canidens Newport, 1844 - новый вид для фауны Кызылординской и Туркестанской областей, a Scutigera coleoptrata (Linnaeus, 1758) - для Акмолинской области. Проиллюстрированы и обсуждены морфологические особенности сколопендровых многоножек. Составлен определительный ключ для мухоловок и сколо- пендровых многоножек Казахстана. Все находки картированы.

\section{Introduction}

To date, three species of scutigeromorph centipedes are known from Kazakhstan: Allothereua kirgisorum Lignau, 1929 (Almaty Region) [Lignau, 1929a, b], Thereuonema turkestana Verhoeff, 1905 (Almaty Region) [Edgecombe, Giribet, 2009], and Scutigera coleoptrata (Linnaeus, 1758) (Kostanay and East Kazakhstan regions) [Dyachkov et al., 2016; Bragina et al., 2020]. The order Scolopendromorpha is represented only by Scolopendra canidens Newport, 1844, from the West Kazakhstan and Mangystau regions [Kessler, 1874; Sseliwanoff, 1884; Zalesskaja, Schileyko, 1991, 1992; Vsevolodova-Perel, 2009; Dyachkov, 2019].

\section{Material and methods}

This paper is based on the material taken by hand and preserved in $70 \%$ ethanol in western, southern and eastern Kazakhstan in 2017-2018 (Fig. 1). The material is shared between the collections of the Altai State University, Barnaul (ASU), the Zoological Museum of the Moscow State University, Moscow (ZMMU), and the Hungarian Natural History Museum, Budapest (HNHM), as indicated below. Some material from the collections of the Perm State University, Perm (PSU) and the Zoological Institute of the Russian Academy of Sciences, Saint Petersburg (ZIN) was also examined. The type material of Thereuonema turkestana Verhoeff, 1905, housed in the Museum für Naturkunde, Berlin (ZMB), was also revised.

The pictures have been taken using an Olympus DP74 digital camera attached to an Olympus SZX16 stereo microscope, Leica DFC490 digital camera attached to a Leica Z16 APO A stereo microscope and a Canon EOS R camera with Canon MP-E 65mm f/2.8 1-5x Macro Photo Lens. The 


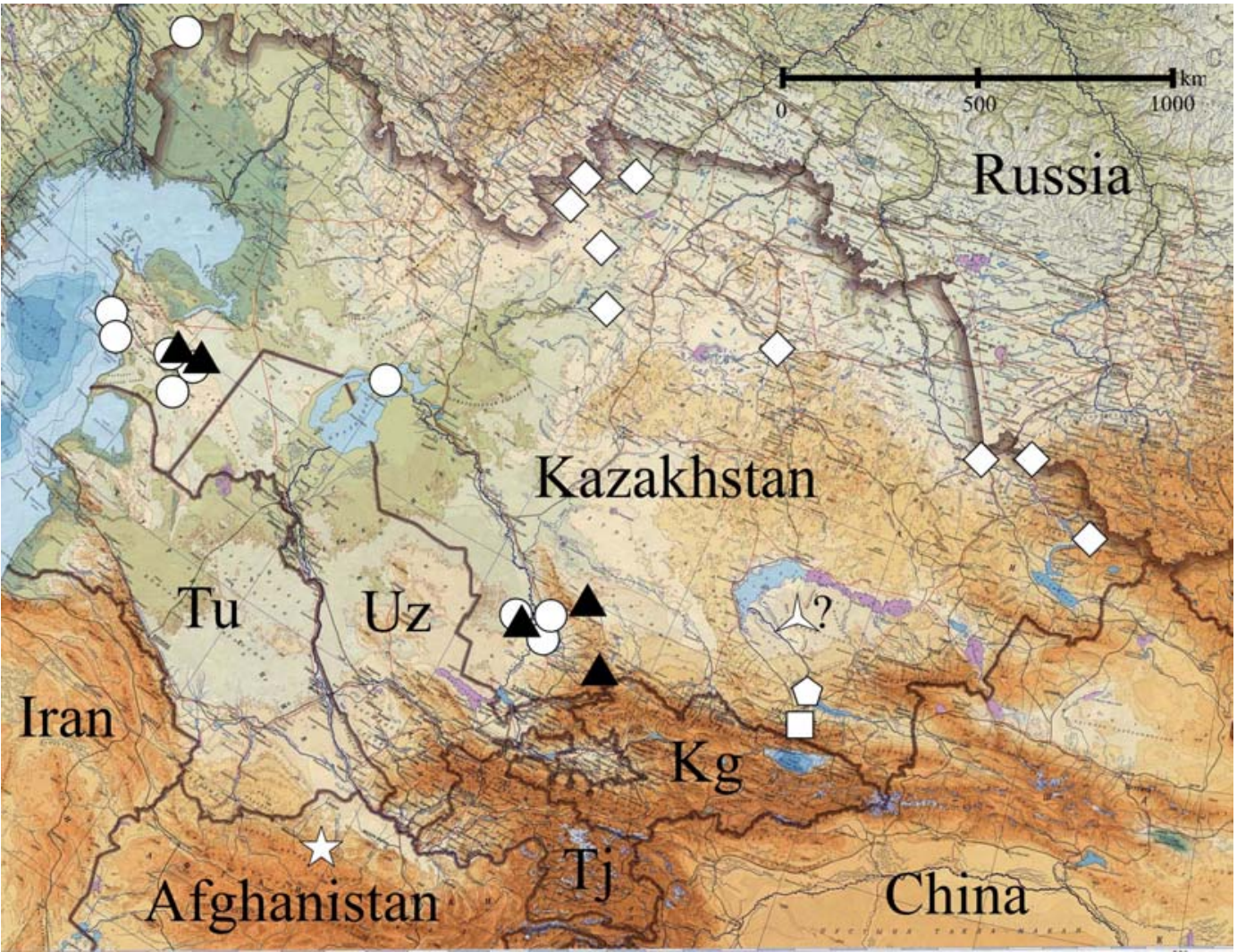

Fig. 1. Distributions of Allotherena kirgisorum Lignau, 1929 - three-pointed star, Scutigera coleoptrata (Linnaeus, 1758) diamond, Cryptops doriae Pocock, 1891 - triangle, C. hortensis (Donovan, 1810) - square, Scolopendra canidens Newport, 1844 circle, Thereuonema turkestana Verhoeff, 1905 — pentagon (in Kazakhstan), C. "afghanus" Loksa, 1971 — star (in Afghanistan); question mark - uncertain locality.

Рис. 1. Распространение видов Allothereua kirgisorum Lignau, 1929 - трехконечная звезда, Scutigera coleoptrata (Linnaeus, 1758) - ромб, Cryptops doriae Pocock, 1891 - треугольник, C. hortensis (Donovan, 1810) - квадрат и Scolopendra canidens Newport, 1844 - круг, Thereuonema turkestana Verhoeff, 1905 - пятиугольник (в Казахстане) и C. «afghanus» Loksa, 1971 звезда (в Афганистане); знак вопроса обозначает неопределенную местность.

images were prepared with the help of Helicon Focus 6.7.1 software. Forcipules and cephalic plates were mounted in permanent slides using euparal.

In the descriptions, the standardized terminology proposed by Bonato et al. [2010] was mainly followed, yet with terms, "spine bristle" and "tergal spiculae", adopted from Edgecombe [2011] and Giribet \& Edgecombe [2013]. Abbreviations: $\mathrm{Tu}$ - Turkmenistan, Uz - Uzbekistan, $\mathrm{Kg}$ Kyrgyzstan, $\mathrm{Tj}$ - Tajikistan, $\mathrm{T}$ - tergite, $\mathrm{S}$ - sternite, $\mathrm{P}$ prefemur, F - femur, Ti - tibia, Ts - tarsus; collectors: AD - A.B. Dairbekova, YD - Yu.V. Dyachkov, EE E.À. Eversmann, F - V. Fet, AF - A.A. Fomichev, YK Yu. Kabanov, DL - D.V. Logunov, YL - Yu.A. Loshkina, KM - K.G. Mikhailov, TVP — T.V. Pavlenko, TP T.S. Perel, DP - D.D. Piriulin, HR - H. Rolle, VR V.V. Rudoi, IT - I.I. Temreshev, TT - T.K. Tuneva; ad. — adults, sad. — subadults, juv. — juveniles, col. — collector. Locality data are given below as on the original labels; additional information is given in square brackets.

\section{Results}

Order Scutigeromorpha Pocock, 1895 Family Scutigeridae Gervais, 1837

\section{Subfamily Thereuoneminae Verhoeff, 1905}

Genus Allothereua Verhoeff, 1905

Type species: Allothereua maculata (Newport, 1844) (by original designation) [Bonato et al., 2016].

\section{Allothereua kirgisorum Lignau, 1929}

Figs 1, 7-9.

Type locality: "Im Sachsaul-Gesträuch (Arthrophytum), beim Tumartscha-Brunnen, ca. $108 \mathrm{~km}$ östlich vom Balchasch-See", according to Lignau [1929a, b]. 23.

Allothereua (?) kirgisorum Lignau, 1929b: 215-217, Abb. 21-

Allothereua (?) kirgisorum — Lignau, 1929a: 174; Edgecombe, 2011: 367.

Allothereua kirgisorum - Dobroruka, 1979: 101-103.

DIAGNOSIS. Even though the original description of $A$. kirgisorum was too succinct and deficient, this species can be recognized because Allothereua differs from Thereuone$m a$ Verhoeff, 1904, the only other genus of Thereuoneminae reported from Central Asia and widely represented there by Thereuonema turkestana Verhoeff, 1905 (eastern Kazakh- 


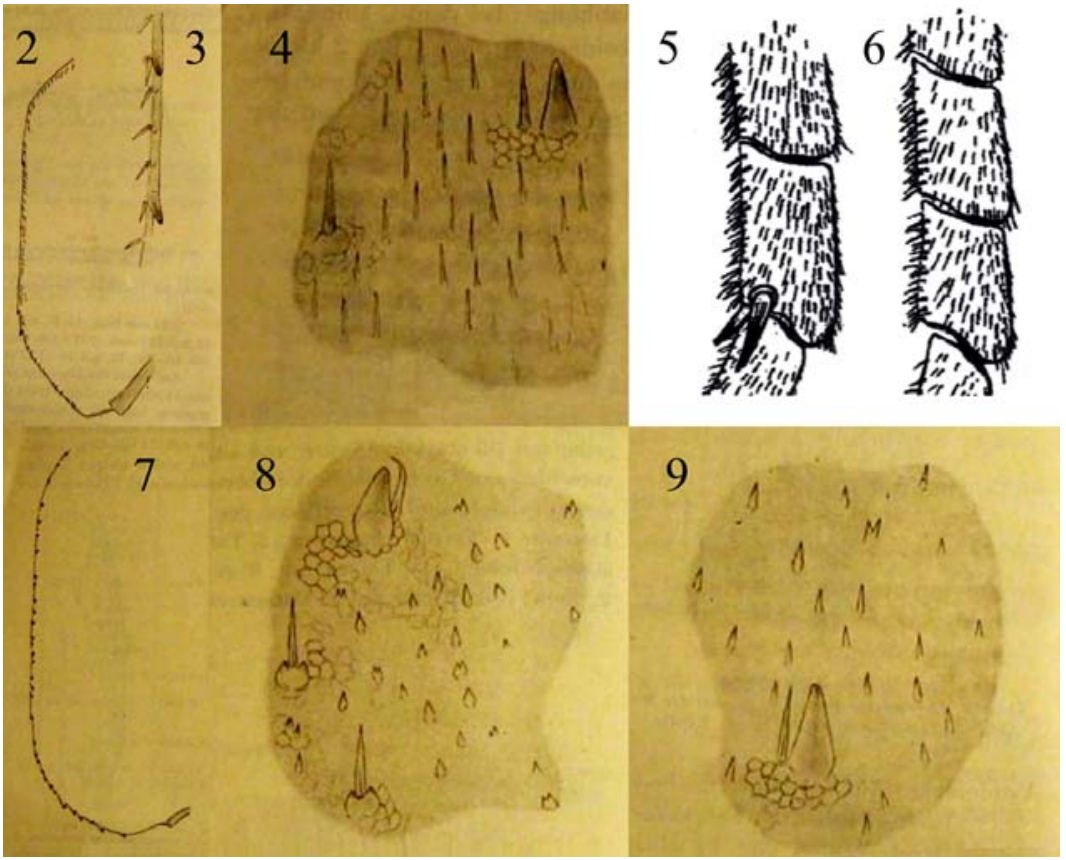

Figs 2-9. Thereuonema turkestana Verhoeff, 1905 (after Lignau [1929b]), dorsal view: 2 - edge of stomatotergite 7; 3 - same, enlarged; 4 - middle part of stomatotergite 7; Scutigera coleoptrata (Linnaeus, 1758) (after Demir [1948]): 5 - distal end of tarsus 1 of legs 6-14, lateral view; Thereuonema microstoma (Meinert, 1886) (after Demir [1948]): 6 - distal end of tarsus 1 of legs 6-14, lateral view; Allotherena kirgisorum Lignau, 1929 (after Lignau [1929b]), dorsal view: 7 - edge of stomatotergite 7; 8 - middle part of stomatotergite 7;9 - fragment of stoma-saddle of tergite 7. Without scale.

Рис. 2-9. Thereuonema turkestana Verhoeff, 1905 (по Lignau [1929b]), сверху: 2 - край тергита 7; 3 - то же, большое увеличение; 4 - средняя часть тергита 7; Scutigera coleoptrata (Linnaeus, 1758) (по Demir [1948]): 5 - дистальный конец лапки 1 ног с 6 по 14 пары, латерально; Thereuonema microstoma (Meinert, 1886) (по Demir [1948]): 6 — дистальный конец лапки 1 ног с 6 по 14 пары, латерально; Allothereua kirgisorum Lignau, 1929 (по Lignau [1929b]), сверху: 7 — край тергита 7; 8 — средняя часть тергита 7; 9 - фрагмент поверхности тергита 7 у стигмы. Без масштаба.

stan, Uzbekistan, Kyrgyzstan and Afghanistan) [Verhoeff, 1905, Lignau, 1929a, b; Würmli, 1975], in the shape of spiculae on the tergites (Figs 2-4, 8-9). The difference between Thereuoneminae and Scutigerinae (Scutigera coleoptrata, known from eastern and central Kazakhstan (see below), and S. asiatica Sseliwanoff, 1884, from the Tashkent Region, Uzbekistan [Sseliwanoff, 1884]) lies in the absence or presence of a pair of spine bristles at the distal end of tarsus 1 of legs $6-14$, respectively (Figs 5, 6). Therefore, only this character has been used in the key below.

REMARKS. This species was described based on a single male collected in 1913 by V.N. Shnitnikov near Tumarcha well in an Arthrophytum grove, $108 \mathrm{~km} \mathrm{E}$ of Lake Balkhash, Balkhash District, Almaty Region. The name Allothereua kirgisorum had first been introduced by Lignau [1929a] as a nomen nudum, without any diagnostic features given. It was only a little later that this species was validated and described [Lignau, 1929b]. Lignau provisionally allocated it in Allothereua, based on the features of spiculae on T6 and T7. No other material has since been reported. Dobroruka [1979] regarded A. kirgisorum as a valid/unquestioned species, but eventually the taxonomic status of this scutigerid remains uncertain and requires revision. There is no information on the whereabouts of the holotype, but anyway it is absent from the ZMMU and ZIN collections. Most probably it was housed in Odessa, where Lignau worked, and is likely to have perished during World War II.
The present location of "Tumarcha well" is not exact, but Shnitnikov [1925: 58; 1934: 20] wrote that "Very nice saxaul [Arthrophytum ammodendron and A. acutifolium] forests are situated southeast of the head of Bakanas, on the road Dzhanay Dzhol; there are the same groves near the place of the divergence of all three Bakanases, near Tumarcha well and in the lower reaches of Bakanas between Korsand Chit-Bakanas". "Three Bakanases" is the name of the former Ili River delta. At the present both Kors-Bakanas and Chit-Bakanas are dry river beds. Even in the years before 1925, as Shnitnikov [1958: 304] wrote, "Today these places cannot be recognized... I visited the head of Bakanas (the dry bed of the river is the former bed of Ili River or one of its beds) in 1910 and 1913. At that time all the place was covered with forests ... The river terrace was partly forested with black saxaul. Now there is a flat steppe... grey clay buildings of the Bakanas Regional Centre are towering over this similarly grey and despondent landscape". Shnitnikov [1928: 27] also mentioned the following localities for some reptiles he had collected: "Between Kiysyk well and Ili River", "between Tumarcha and Kiysyk wells", "Bakanas near Tumarcha well". Tumarcha well thus seems to have been located in the region near Ili River and the dry Bakanas bed. As I was unable to relocate any Tumarcha or Kiysyk well on detailed Soviet topographic military maps (also known as Genshtab maps) of the Almaty Region, I am inclined to consider the type locality of $A$. kirgisorum as uncertain, lying somewhere within the Balkhash District of the Almaty Region. 


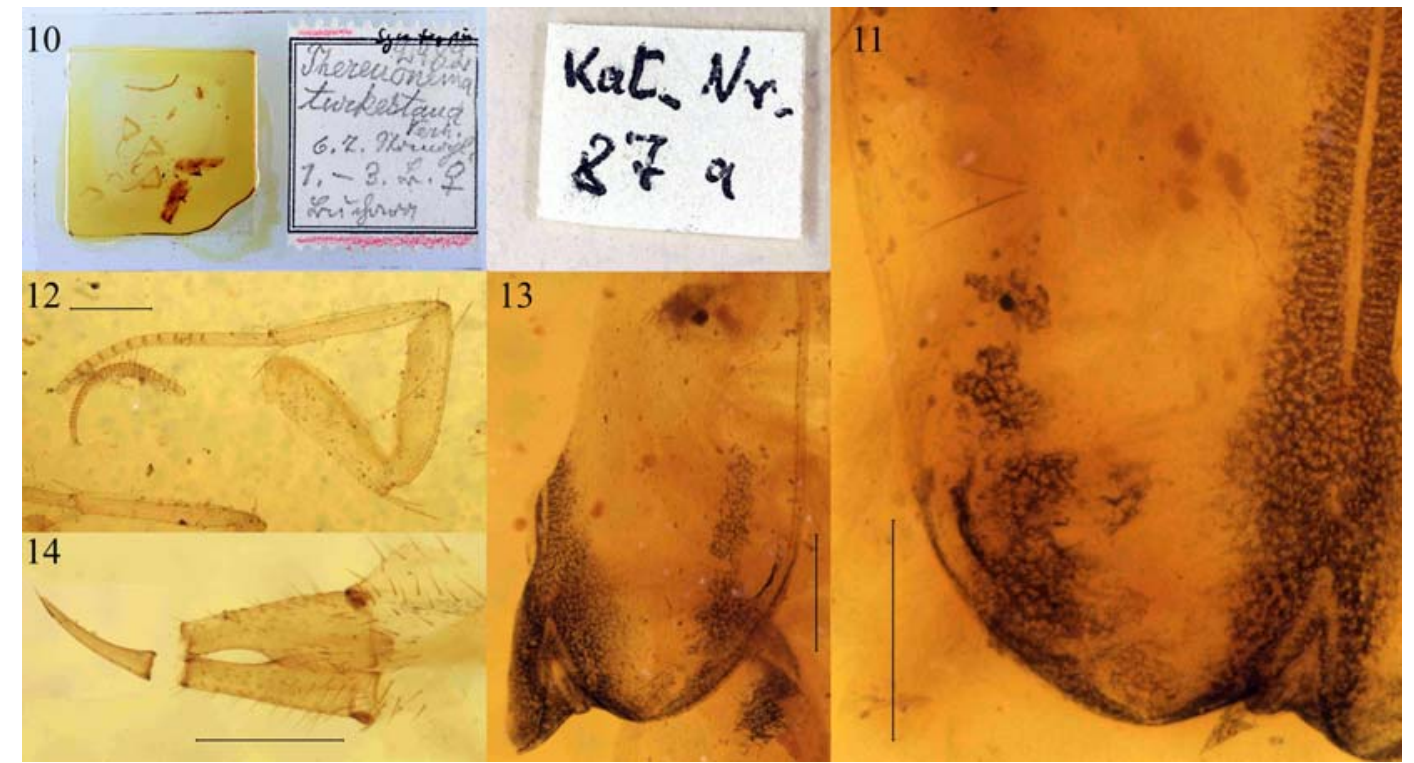

Figs 10-14. Thereuonema turkestana Verhoeff, 1905 (ZMB 87a, female): 10 - microscopic slide from Karl Verhoeff's collection; 11 left part of stomatotergite 7, dorsal view; 12 - leg 2, lateral view; 13 - right part of stomatotergite $6 ; 14$ - gonopods, ventral view. Scale: $0.1 \mathrm{~mm}(12), 0.5 \mathrm{~mm}(11,13,14)$.

Рис. 10-14. Thereuonema turkestana Verhoeff, 1905 (ZMB 87a, самка): 10 - слайд из коллекции Карла Фирхофа; 11 - левая часть тергита 7, сверху; 12 - нога 2, латерально; 13 - правая часть тергита 6; 14 - гоноподы, вентрально. Масштаб: 0,1 мм (12), 0,5 мм $(11,13,14)$.

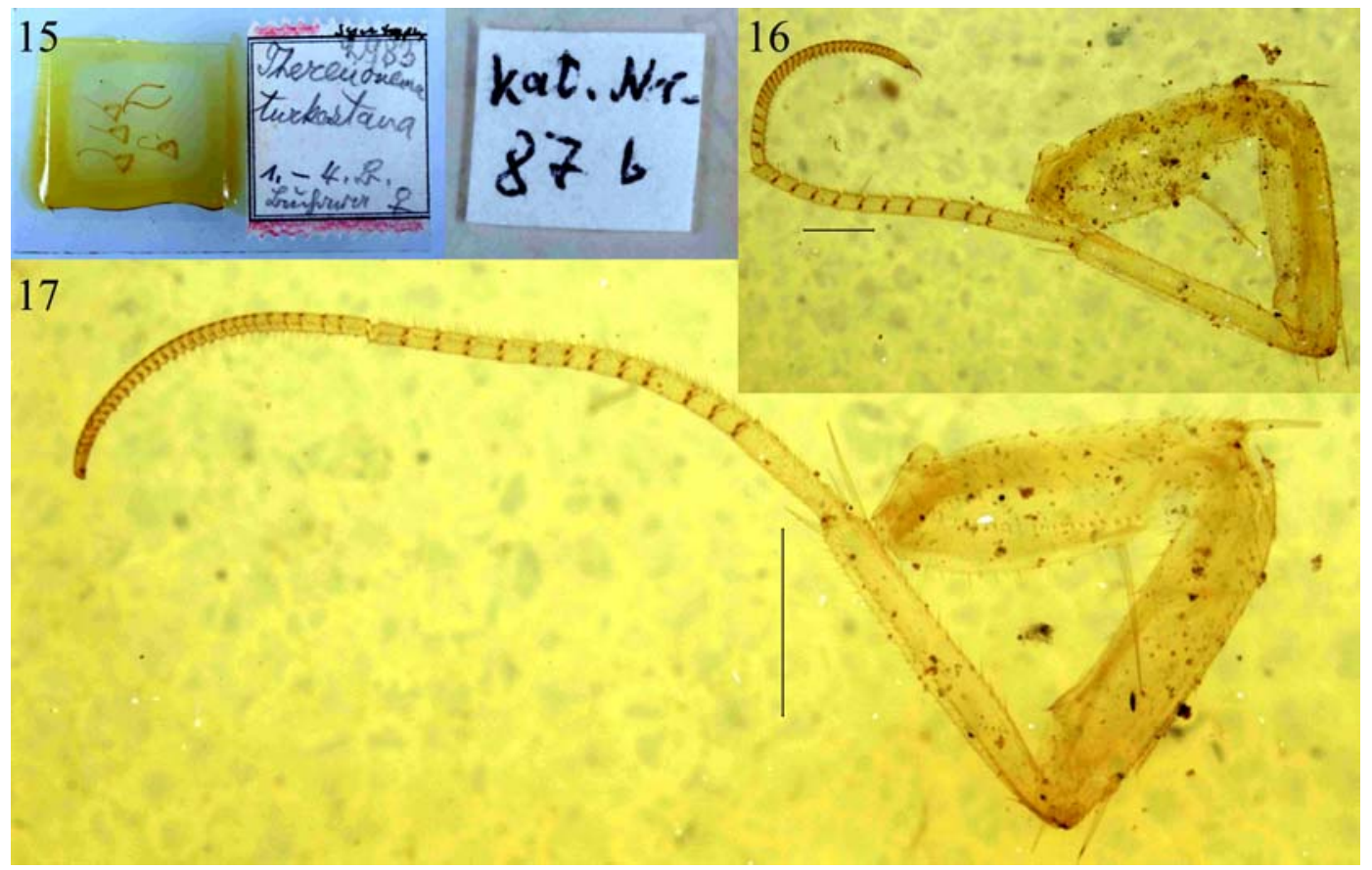

Figs 15-17. Therenonema turkestana Verhoeff, 1905 (ZMB 87b, female): 15 - microscopic slide from Karl Verhoeff's collection; 16 leg 3, lateral view; 17 - leg 4, lateral view. Scale: $0.5 \mathrm{~mm}(16), 1 \mathrm{~mm}(17)$.

Рис. 15-17. Thereuonema turkestana Verhoeff, 1905 (ZMB 87b, самка): 15 - слайд из коллекции Карла Фирхофа; 16 - нога 3 , сбоку; 17 - нога 4, сбоку. Масштаб: 0,5 мм (16), 1 мм (17).

\section{Genus Thereuonema Verhoeff, 1904}

Type species: Cermatia tuberculata Wood, 1862 (by subsequent designation) [Bonato et al., 2016].
Thereuonema turkestana Verhoeff, 1905 Figs 1-4, 10-25.

Locus typicus: "Buchara", Uzbekistan [Verhoeff, 1905]. Thereuonema turkestana Verhoeff, 1905: 88-89. 


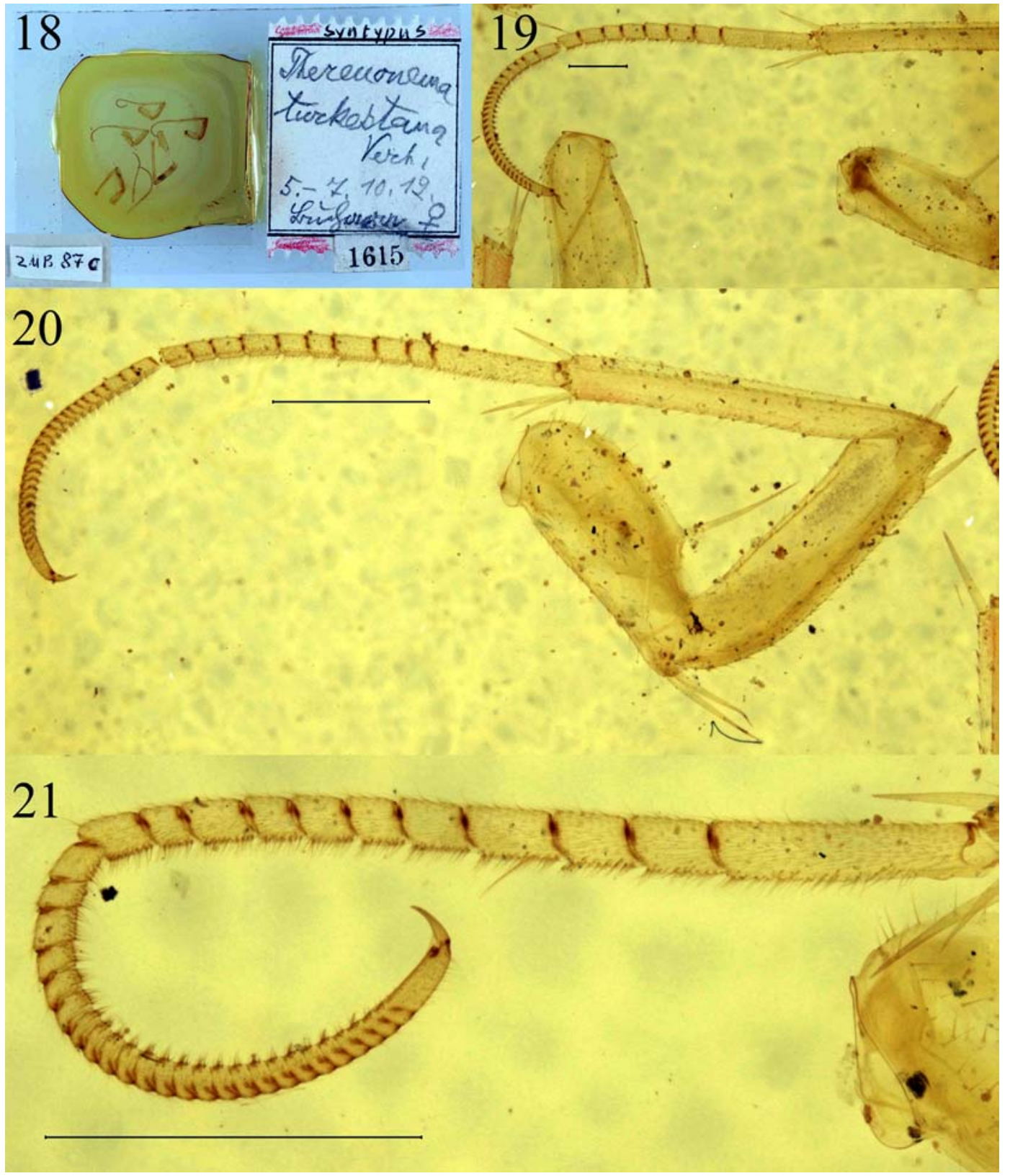

Figs 18-21. Thereuonema turkestana Verhoeff, 1905 (ZMB 87c, female): 18 - microscopic slide from Karl Verhoeff's collection; 19 tarsus of leg 7, lateral view; 20 - tarsus of leg 6, lateral view; 21 - tarsus of leg 5, lateral view. Scale: $0.5 \mathrm{~mm}(19), 1 \mathrm{~mm}(20,21)$.

Рис. 18-21. Thereuonema turkestana Verhoeff, 1905 (ZMB 87c, самка): 18 - слайд из коллекции Карла Фирхофа; 19 - лапка ноги 7, сбоку; 20 - лапка ноги 6, сбоку; 21 - лапка ноги 5, сбоку. Масштаб: 0,5 мм (19), 1 мм (20, 21).

Thereuonema turkestana — Lignau, 1929a: 174; 1929b: 211214; Stoev, 2002: 82; Edgecombe, Giribet, 2009: 421; Edgecombe, 2011: 367; Dunlop et al., 2017: 290.

Thereuonema oreiotes Lignau, 1929b: 214-215.

Thereuonema oreiotes — Würmli, 1975: 195.

MATERIAL. Syntypes: 3 of (ZMB 87a-c), Uzbekistan, Buchara, [1820], col. EE; $3 \sigma^{\top} \sigma^{\top}, 3$ OO (ZMB unnumbered), Kyrgyzstan? [see Remarks], Alexandrowskaja Gebirge [now Kyrgyz Ala-Too Mt. Range], Kappak-District, 5./V.[19]06, col. HR.

DIAGNOSIS. Tergal spiculae long, needle-shaped (tapering) (Fig. 4); 160-170 isolated bristles on tergite 6 [Verhoeff, 1905; Edgecombe, 2011].
DISTRIBUTION. Afghanistan: Kabul; N India: Kargi [Stoev, 2002]; Uzbekistan: Bukhara, Samarqand and Tashkent regions; Tajikistan: Sughd Region (Khujand); Kyrgyzstan: Naryn (Song Kol Lake) and Chuy (Kyrgyz Ala-Too Mt Range) regions [Lignau, 1929a, b; Würmli, 1975; Dunlop et al., 2017]; Kazakhstan: Almaty Region, Talgar District [Edgecombe, Giribet, 2009].

REMARKS. I have been unable to relocate the present place of the "Kappak-District" on Kyrgyz Ala-Too Mt. Range, but this range lies mostly in Kyrgyzstan. 

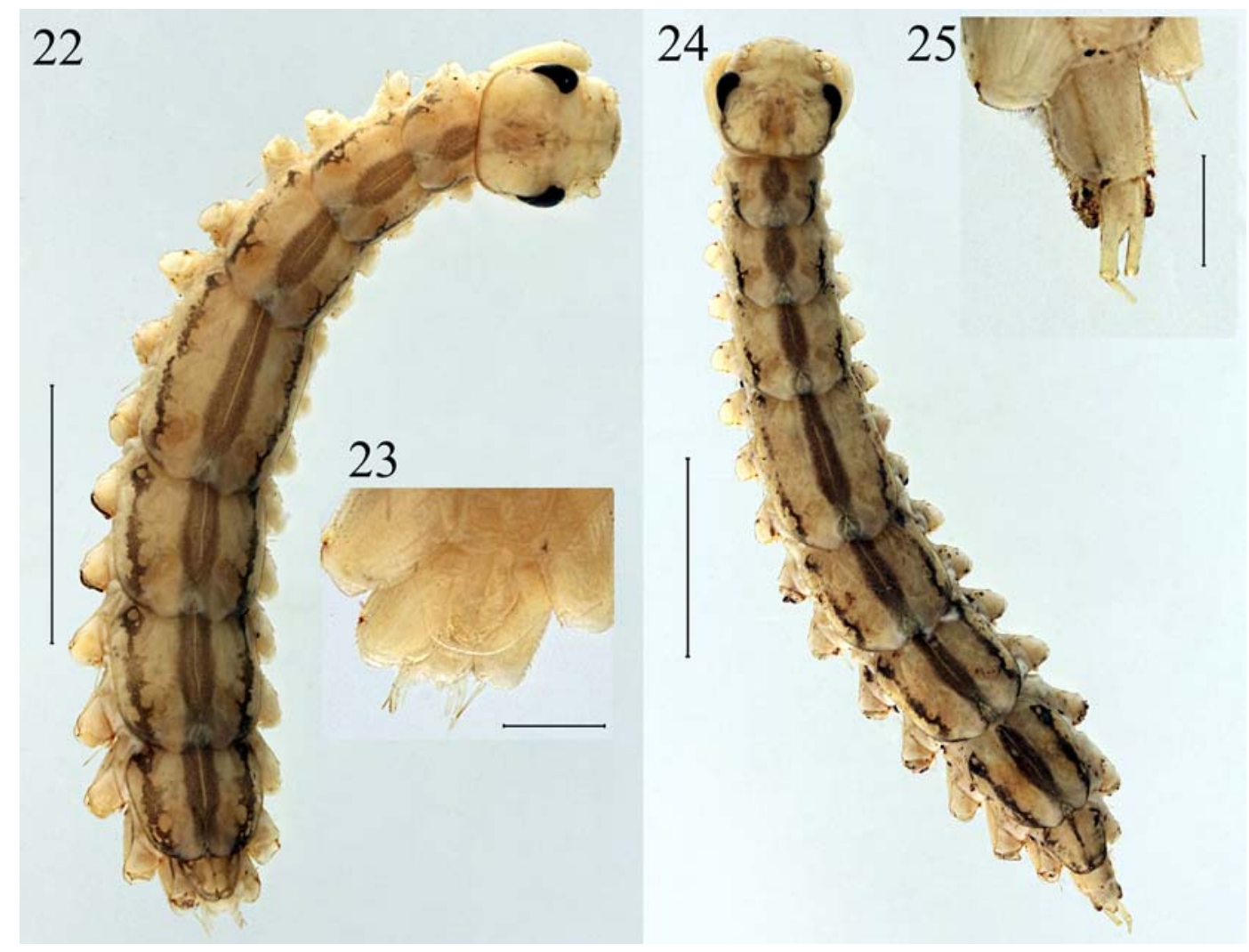

Figs 22-25. Thereuonema turkestana Verhoeff, 1905 (non-type, ZMB unnumbered, Kyrgyzstan): 22 - habitus of male, dorsal view; 23 - male gonopods, ventral view; 24 - habitus of female, dorsal view; 25 - female gonopods, ventral view. Scale: $1 \mathrm{~mm}(23,25), 5 \mathrm{~mm}$ $(22,24)$.

Pис. 22-25. Thereuonema turkestana Verhoeff, 1905 (нетиповой материал, ZMB без номера, Киргизия): 22 - габитус, самец, сверху; 23 - гоноподы самца, снизу; 24 - габитус, самка, сверху; 25 - гоноподы самки, снизу. Масштаб: 1 мм (23, 25), 5 мм (22, 24).

Subfamily Scutigerinae Leach, 1814

Genus Scutigera Lamarck, 1801

Type species: Scolopendra coleoptrata Linnaeus, 1758 (by original designation) [Bonato et al., 2016].

\section{Scutigera coleoptrata (Linnaeus, 1758)}

Figs 1, 5.

Terra typica: Spain [Bonato et al., 2016].

Scutigera coleoptrata - Stoev, Geoffroy, 2004: 4; Faúndez, 2011: 336; Dyachkov et al. 2016: 440; Nefediev et al., 2016a: 428-431; Ostrovsky, 2016: 455; Zuev, 2016: 23; Zuev, Evsyukov, 2016: 417; Bragina et al., 2020: 28.

MATERIAL. Kazakhstan, East Kazakhstan Region: 1 ( (ASU No. 157), Semey City, inside a house, 10.VI.2018, col. YL; $10^{7}$ (ASU No. 158), inside a house, 19.IX.2018, col. AD; 1 (ASU No. 163), Shemonaikha District, Shemonaikha Town, garden, 6.V.2017, col. VR; Akmola Region: 1 (ASU No. 149), Astana [Nur-Sultan] City, Chubary District, 16.VI.2017, col. IT.

DISTRIBUTION. This widespread synanthropic species occurring in Europe (including the Urals in the European part of Russia), western Siberia, western, Central and East Asia, Africa; also recorded from Australia, North and South America [Lewis, 1981; Stoev, Geoffroy, 2004; Farzalieva, 2008; Faúndez, 2011; Volkova, 2014; Zuev, 2016; Nefediev et al., 2016a; Ostrovsky, 2016]. Kazakhstan: East Kazakh- stan [Dyachkov et al., 2016], Kostanay [Bragina et al., 2020] and Akmola regions.

REMARKS. S. coleoptrata is new to the fauna of the Akmola Region. In Kazakhstan, this species dwells mostly indoors.

Order Scolopendromorpha Leach, 1815

Family Cryptopidae Kohlrausch, 1881

Genus Cryptops Leach, 1814

Type species: Scolopendra hortensis Donovan, 1810 (by monotypy) [Bonato et al., 2016].

\section{Cryptops (Cryptops) doriae Pocock, 1891 Figs 1, 26-29.}

Locus typicus: "Lectotype from Burma (= Myanmar): Palon. Two other (missing?) syntypes from Shwegoo and Chiala, also in Myanmar" [Schileyko, 2007].

Cryptops doriae - Barber, 2009: 128.

Cryptops (Cryptops) doriae - Attems, 1930: 214; Lewis, 2007: 12, 2013: 12; Schileyko, 2007: 86; Tran et al., 2013: 220; Schileyko, Stoev, 2016: 262.

MATERIAL. Kazakhstan, Mangystau Region, Ustyurt Nature Reserve, western chink of Ustyurt Plateu: 1 ad. (ASU No. 203), near Mamekkazgan cordon, stony foothill of a chink (cliff), under 


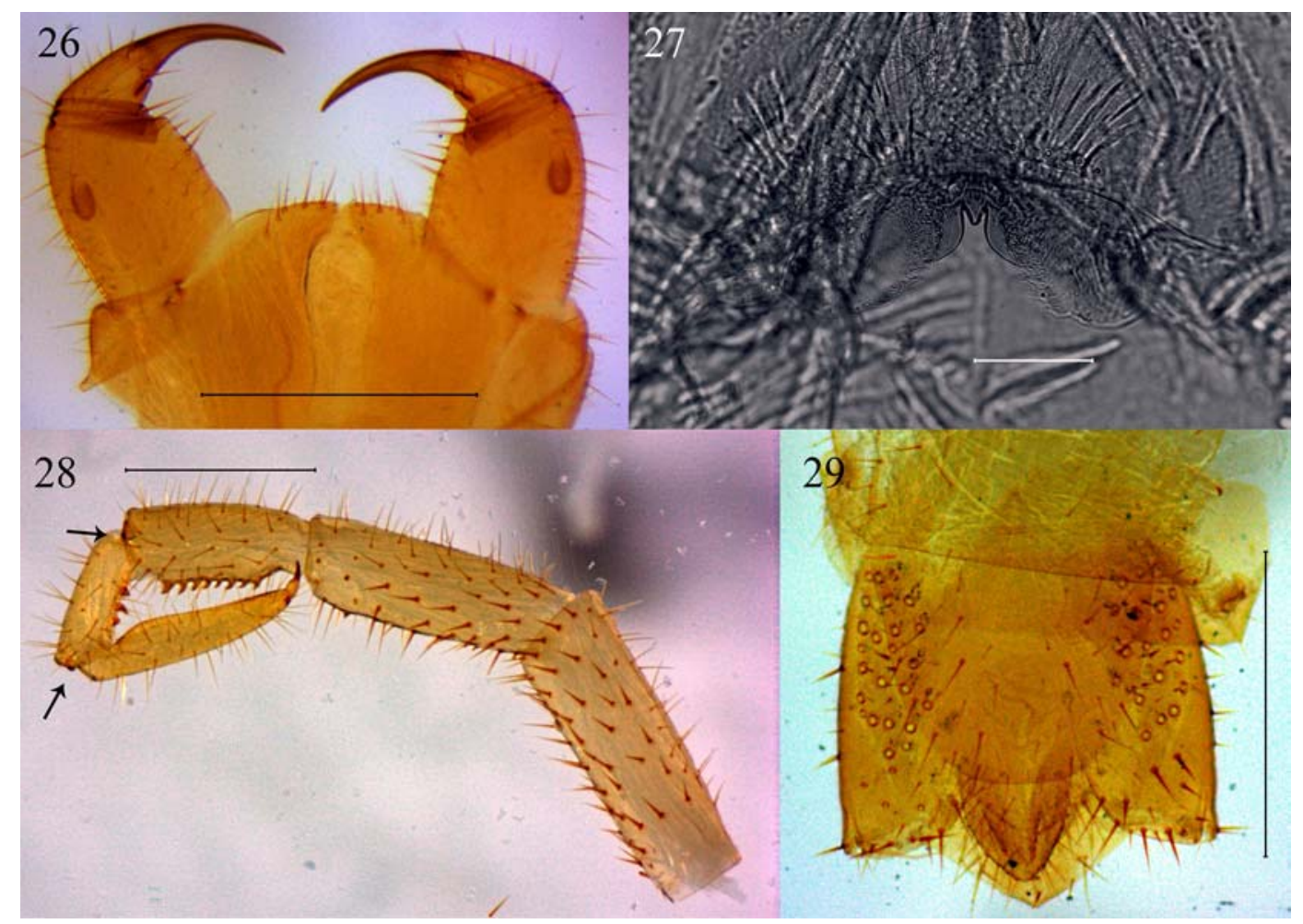

Figs 26-29. Cryptops doriae Pocock, 1891 (26-27, 29 - ASU No. 205, 28 - ASU No. 204): 26 - forcipular segment, ventral view; 27 - clypeus, ventral view; 28 - ultimate leg, lateral view; 29 - terminal part of body, ventral view. Scale: $0.5 \mathrm{~mm}(26,28), 0.1 \mathrm{~mm}$ (27), $0.25 \mathrm{~mm}(29)$.

Рис. 26-29. Cryptops doriae Pocock, 1891 (26-27, 29 - ASU No. 205, 28 - ASU No. 204): 26 - ногочелюстной сегмент, снизу; 27 - клипеус, снизу; 28 - нога последней пары, сбоку; 29 - задняя часть тела, снизу. Масштаб: 0,5 мм (26, 28), 0,1 мм (27), 0,25 мм (29).

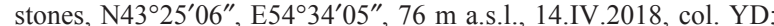
1 ad. (HNHM chilo-7684), stony foothill of a chink, $76 \mathrm{~m}$ a.s.1., 12-13.IV.2018, col. AF; 3 ad. (ASU No. 205), between Karpakkazgan and Karzhar wells, stony foothill of a chink, under stones,

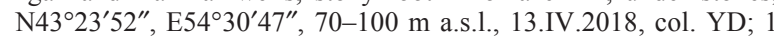
sad. (ASU No. 206), near Mt Baszhira, chink, under stones, N43 22'42", E54 $07^{\prime} 25^{\prime \prime}, 150 \mathrm{~m}$ a.s.1., 15.IV.2018, col. YD; 1 ad., 1 juv. (ASU No. 207), near Kokosim well, under stones, N43 $10^{\prime} 12^{\prime \prime}$, E54 ${ }^{\circ} 2^{\prime} 40^{\prime \prime}, 270 \mathrm{~m}$ a.s.1., 14.IV.2018, col. YD; 1 ad. (HNHM

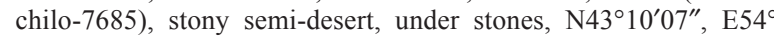
52'49", 250 m a.s.1., 14.IV.2018, col. AF; Turkistan Region: 1 ad. (ZMMU Rc 7713), $50 \mathrm{~km} \mathrm{NW} \mathrm{Achisay} \mathrm{Village,} \mathrm{shore} \mathrm{of} \mathrm{Lake}$ Kyzylkol, in clay stones, $\mathrm{N}^{\circ} 3^{\circ} 46^{\prime} 34.0^{\prime \prime}$, E69 $30^{\prime} 36.4^{\prime \prime}, 328 \mathrm{~m}$ a.s.1., 8-9.V.2017, col. YD; 3 ad. (ZMMU Rc 7714), Kayraktau Mts, ravines, dry steppe, $\mathrm{N} 42^{\circ} 17^{\prime} 53.8^{\prime \prime}, \mathrm{E} 67^{\circ} 46^{\prime} 21.0^{\prime \prime}, 314 \mathrm{~m}$ a.s.1., 28.IV.2017, col. YD; 1 ad. (ZMMU Rc 7715), Western Tian-Shan Mts, Ugam Mt Range, Syaram-Ugam National Park, 10 km NE Tylkubas Village, Iirsu River valley, meadow, under stones, N42 24'58.0", E70 21'30.08", 1296 m a.s.l., 16-18.V.2017, col. YD.

DESCRIPTION. Body length $12-23 \mathrm{~mm}$; colour yellow. Posterior margin of head covered by T1. Antennae consisting of 17 segments (rarely $15+17$ or $15+16$ ). Labrum with a single median tooth (Fig. 27). Anterior margin of forcipular coxosternite slightly bisinuate, with 3-4 marginal setae and 2-4 long plus 2-7 minute submarginal ones (Fig. 26). Incomplete posterior paramedian sutures from $T 3$, complete ones plus lateral sutures from T4. Ultimate $\mathrm{T}$ with a well-developed longitudinal depression posteriorly. Ultimate $\mathrm{S}$ trapeziform, with rounded posterior margin (Fig. 29). Anterior two-thirds of coxopleural surface occupied by an oval pore field consisting of 13-25 pores of various sizes. All legs with flexible tarsal articulations. Saw teeth of ultimate leg: F with one tooth, Ti with 8 teeth and Ts 1 with 3-4 teeth in specimens from the Mangystau Region; $F$ with one tooth, Ti with 6 teeth and Ts1 with 3 teeth in individuals from Turkistan Region; distal spiny process present on $\mathrm{Ti}$ and Ts1 (Fig. 28).

DISTRIBUTION. Afrotropical (Seychelles), Indo-Malayan (Cambodia, Laos, Myanmar, India, Nepal, Vietnam, Java) and Australian (Papua New Guinea) regions [Attems, 1930; Lewis, 1999, 2013; Schileyko, 2007; Tran et al., 2013]. Palaearctic Region: Central Asia (Kazakhstan); also introduced to Great Britain (see Lewis [2007]; Barber [2009]).

REMARKS. This species is presently recorded from the Palaearctic Region for the first time (except for a botanical garden in Great Britain), also being new to the Kazakhstan fauna. The specimens studied agree well with the description of the dubious $C$. (C.) afghanus Loksa, 1971 (see Loksa [1971]) from Afghanistan (Fig. 1), which is a nomen dubi$u m$, according to Lewis [2013: 4].

\section{Cryptops (Cryptops) hortensis (Donovan, 1810)} Figs 1, 30-33.

Type locality: "gardens at Exeter", United Kingdom [Donovan, 1810]. 475.

Scolopendra hortensis Donovan, 1810: 23, Figure on Plate 


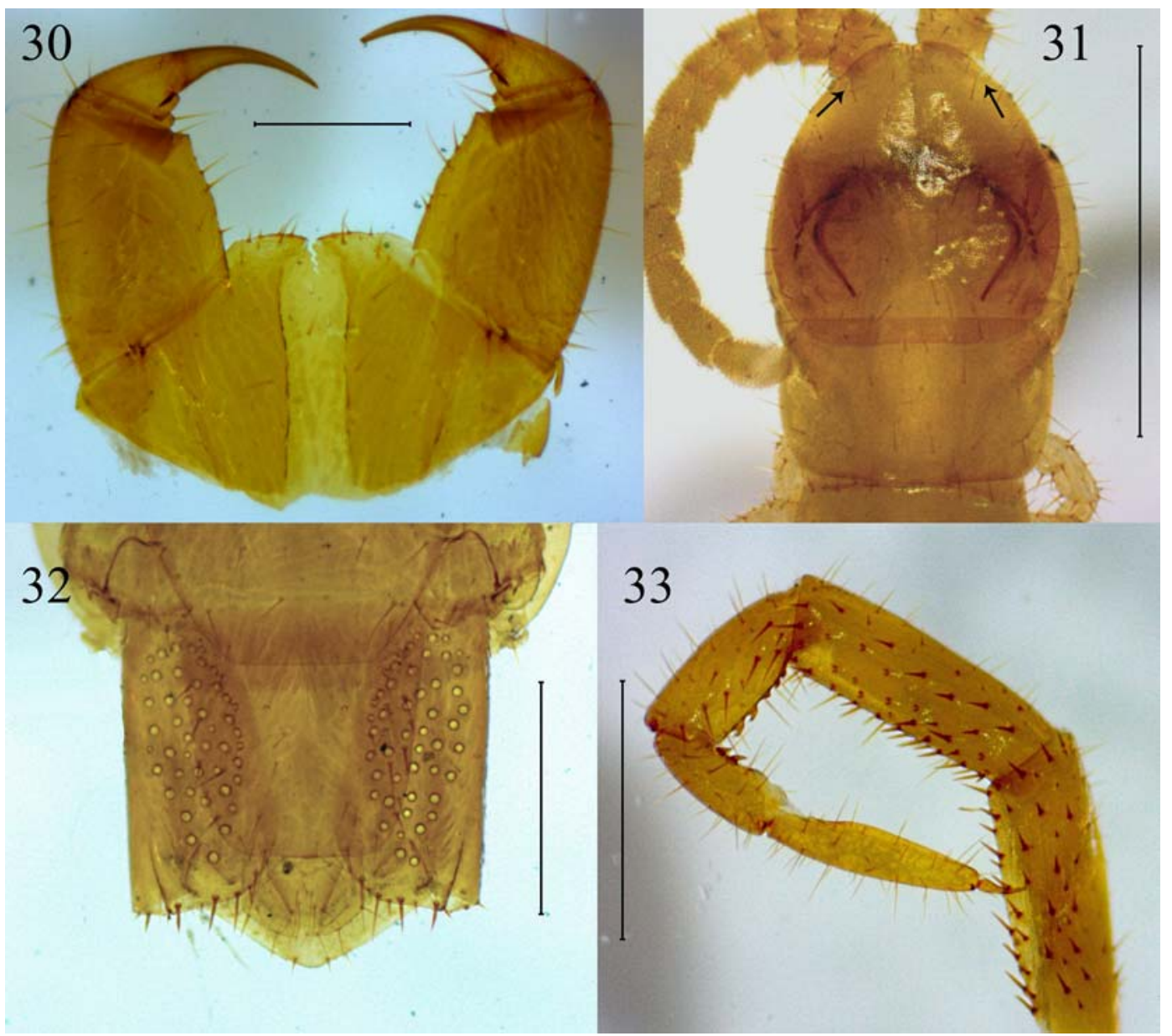

Figs 30-33. Cryptops hortensis (Donovan, 1810) (30, 32 - ASU No. 210; 31, 33 - ASU No. 213): 30 - forcipular segment, ventral view; 31 - head and tergite 1, dorsal view; 32 - terminal part of body, ventral view; 33 - ultimate leg, lateral view. Scale: $0.25 \mathrm{~mm}(30)$, $1 \mathrm{~mm}(31), 0.5 \mathrm{~mm}(32,33)$.

Рис. 30-33. Cryptops hortensis (Donovan, 1810) (30, 32 - ASU No. 210; 31, 33 - ASU No. 213): 30 - ногочелюстной сегмент, снизу; 31 - голова и тергит 1 , сверху; 32 - задняя часть тела, снизу; 33 - нога последней пары, сбоку. Масштаб: 0,25 мм (30), 1 мм (31), 0,5 мм $(32,33)$.

Cryptops hortensis — Zalesskaja, Schileyko, 1992: 370; Barber, 2009: 124.

Cryptops (Cryptops) hortensis - Attems, 1930: 207; Zalesskaja, Schileyko, 1991: 24; Lewis, 2011: 24; Nefediev et al., 2016b: 107; Zuev, 2016: 31.

MATERIAL. Kazakhstan, Almaty Region, Almaty City, Rakhat District, thicket with grass and fruit trees, under stones, planks, col. IT: 1 ad. (ASU No. 209), 30.X.2018; 1 ad. (ASU No. 210), 28.III.2018; 2 ad. (ASU No. 211), 7.XI.2018; 3 ad. (ASU No. 212), 5-6.IX.2018; 3 ad. (ASU No. 213), 29-30.III.2018; 2 ad. (ASU No. 219), 26.III.2018; 1 ad. (ASU No. 220), 27.III.2018.

DESCRIPTION. Body length up to $20 \mathrm{~mm}$, dark yellow or brown. Cephalic plate elongated, with paramedian sutures strongly shortened anteriorly (Fig. 31). Antennae with 17 segments. Anterior margin of forcipular coxosternite slightly convex, with 4-7 marginal and 2-4 submarginal ones (Fig. 30). Posterior margin of S21 straight (Fig. 32). Coxopleural pore fields with $30-45$ pores. Saw teeth of ultimate legs: Ti with 5-8, Ts with 2-4 teeth (Fig. 33).

DISTRIBUTION. Widespread in Europe, North Africa (Morocco), western Asia (Georgia, Armenia, Azerbaijan), Central Asia (Tajikistan, Uzbekistan, Turkmenistan), introduced to the United States, Canada, St. Helena Island; Aus- tralia (Tasmania); recorded from synanthropic habitats in Sweden, Finland and western Siberia [Zalesskaja, Schileyko, 1991, 1992; Barber, 2009; Lewis, 2011; Zuev, 2016; Nefediev et al., 2016b; Ostrovsky, 2018].

REMARKS. The specimens studied agree well with the descriptions of Zalesskaja and Schileyko [1991], Barber [2009] and Lewis [2011]. This species is new to the fauna of Kazakhstan, recorded only from anthropogenic habitats.

Family Scolopendridae Leach, 1814 Genus Scolopendra Linnaeus, 1758

Type species: Scolopendra morsitans Linnaeus, 1758 (by original designation) [Bonato et al., 2016].

\section{Scolopendra canidens Newport, 1844 Figs 1, 34-37.}

Terra typica: "Egypt" [Bonato et al., 2016].

Scolopendra aralocaspia Kessler, 1874: 33-37; Sseliwanoff, 1884: $115-116$ 


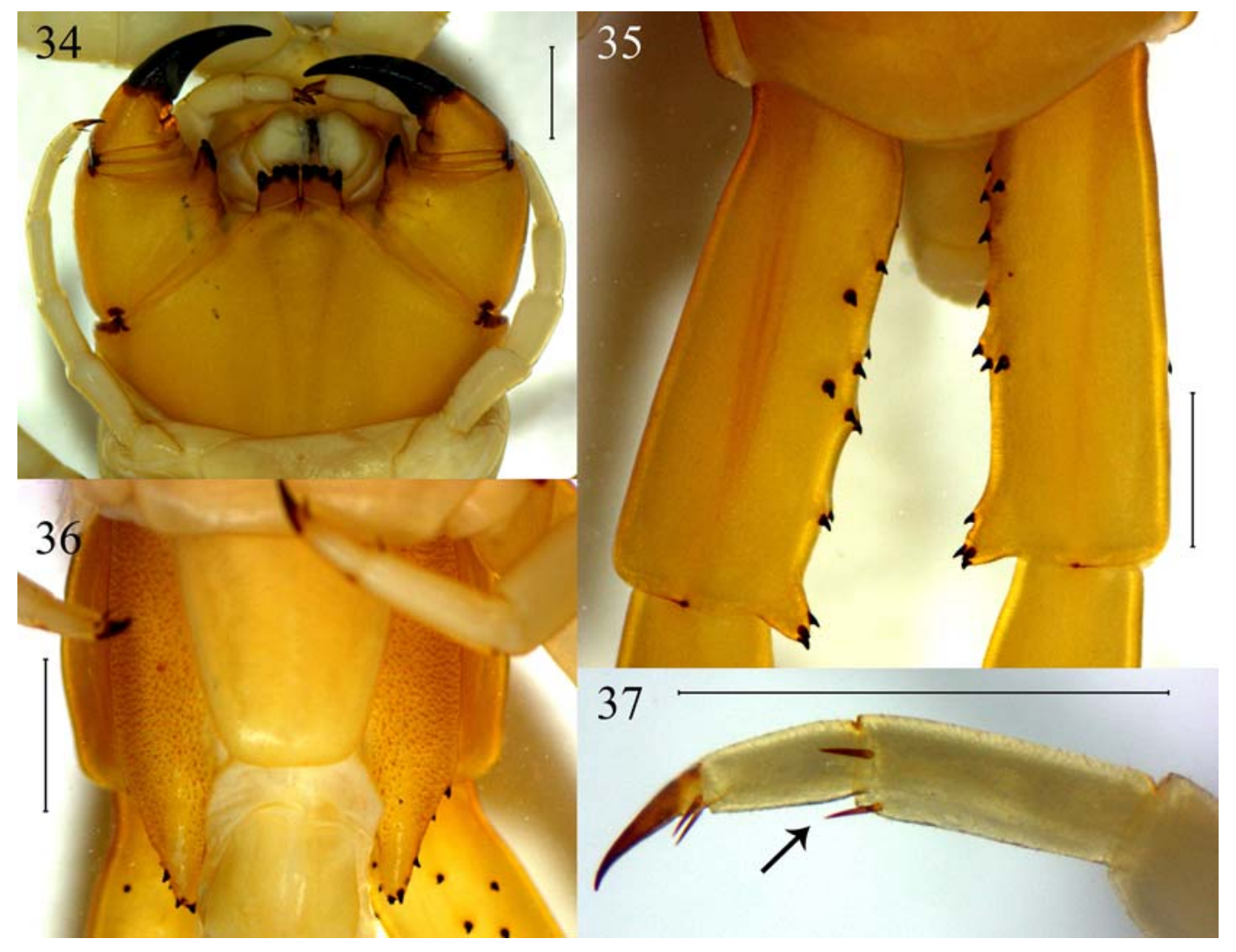

Figs 34-37. Scolopendra canidens Newport, 1844 (ASU No. 191): 34 - forcipular segment, ventral view; 35 - prefemora of ultimate legs, dorsal view; 36 - ultimate leg-bearing segment, ventral view; 37 - tarsus + pretarsus of leg 1, lateral view. Scale: $1 \mathrm{~mm}$.

Рис. 34-37. Scolopendra canidens Newport, 1844 (ASU No. 191): 34 - ногочелюстной сегмент, снизу; 35 - базальные членики ног последней пары, сверху; 36 - задняя часть тела, снизу; 37 - лапка первой пары ног, сбоку. Масштаб: 1 мм.

Scolopendra aralocaspica (sic) - Attems, 1904: 115.

Rhadinoscytalis canidens - Lignau, 1929a: 167

Scolopendra canidens - Kraepelin, 1903: 248; Attems, 1930: 35; Zalesskaja, Schileyko, 1991: 11; 1992: 370; Lewis, 2001: 151; Akkari et al., 2008: 80.

MATERIAL. Kazakhstan, Mangystau Region, Ustyurt Nature Reserve, western chink of Ustyurt Plateau: 9 ad. (ASU No. 179),

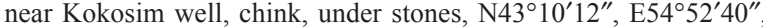
$270 \mathrm{~m}$ a.s.l., 4.IV.2018, col. YD; 1 juv. (ASU No. 193), chink, under stones, $\mathrm{N}^{\circ} 3^{\circ} 10^{\prime} 12^{\prime \prime}$, E54 ${ }^{\circ} 52^{\prime} 40^{\prime \prime}, 270 \mathrm{~m}$ a.s.l., 14.IV.2018, col. YD; 9 ad. (HNHM chilo-7667), between Karpakkazgan and Karzhar wells, stony foothill of a chink (cliff), under stones,

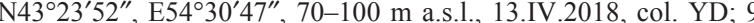
ad. (HNHM chilo-7668), col. YD; 2 ad. (ASU No. 182), SW part of Karynzharyk Depression, near Unere well, stony desert, under stones, N42 ${ }^{\circ} 36^{\prime}$, E54 $04^{\prime}$, 0-5 $\mathrm{m}$ a.s.1., 8.IV.2018, col. YD; 1 ad. (ASU No. 183), S part of Karynzharyk Depression, near Mt Kyzylsay, stony desert with bushes and rocks, N42 $34^{\prime} 57^{\prime \prime}$, E5 $54^{\circ} 13^{\prime} 56^{\prime \prime},-30$ - 30 m a.s.1., 9.IV.2018, col. YD; 1 ad. (HNHM chilo-7670), near Mt Kyzylsay, stony desert with bushes and rocks, N42 $34^{\prime} 57^{\prime \prime}$, E54 $4^{\circ} 13^{\prime} 56^{\prime \prime},-30-30$ m a.s.1., 9.IV.2018, col. YD; 6 ad. (ASU No 202 ), stony desert with bushes and rocks, N42 $34^{\prime} 57^{\prime \prime}$, E $54^{\circ} 13^{\prime} 56^{\prime \prime}$, $-30-30$, 9.IV.2018, col. YD, AF; 1 ad., 1 juv. (HNHM chilo7671), SW part of Karynzharyk Depression, near Unere well, stony desert with bushes, $\mathrm{N}^{\circ} 2^{\circ} 36^{\prime}$, E54 $09^{\circ}$, 0-37 m a.s.l., 8-11.IV.2018, col. AF; 1 juv. (HNHM chilo-7665), col. AF; 2 ad. (ASU No. 191), stony desert with bushes, $\mathrm{N} 42^{\circ} 36^{\prime}, \mathrm{E} 54^{\circ} 09^{\prime}, 0-37 \mathrm{~m}$ a.s.l., 8.IV 2018, col. AF; 6 juv. (ASU No. 185), between Karpakkazgan and Karzhar wells, stony foothill of a chink (cliff), under stones,

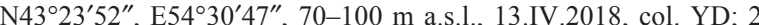
juv. (ASU No. 194), near Mamekkazgan well, stony foothill of a

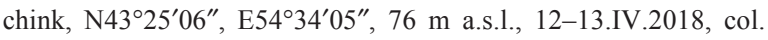
YD; 2 juv. (HNHM chilo-7674), near Elshibek well, foothill of chink, under stones, $\mathrm{N} 42^{\circ} 38^{\prime} 10^{\prime \prime}, \mathrm{E} 54^{\circ} 20^{\prime} 04^{\prime \prime},-15 \mathrm{~m}$ a.s.l., 11.IV.2018, col. YD; 1 juv. (HNHM chilo-7673), stony foothill of a chink, under stones, $\mathrm{N} 43^{\circ} 25^{\prime} 06^{\prime \prime}, \mathrm{E} 54^{\circ} 34^{\prime} 05^{\prime \prime}, 76 \mathrm{~m}$ a.s.1., 12 14.IV.2018, col. YD; eastern bottom of Caspian Sea: 3 ad. (ZMMU Rc 6022), between Sachandyk and Karamandy capes, 13.V.1966, col. YK; 1 ad. (ZMMU Rc 6102), southern Ustyurt, in clay, 3.V.1985; 3 ad. (ZMMU Rc 6109), Mangyshlak, 9.V.1966, col. F; 4 ad. (ZMMU Rc 6144), Aktau City, wells, 10.V.1966; 4 ad. (ZMMU Rc 6152), Sagandyk Cape, 11.V.1966, col. YK; Turkistan Region: 3 ad. (ASU No. 186), Kyzylkum Desert, $100 \mathrm{~km} \mathrm{~W}$ of Arys Town, Kayraktau Mts, grasses, Haloxylon, N42 $20^{\prime} 55.0^{\prime \prime}$, E67 38'19.4", 222 m a.s.1., 1.V.2017, col. YD; 1 ad. (ZMMU Rc 7717), steppe, ravines, in clay and sand, $292 \mathrm{~m}$ a.s.1., 2.V.2017, col. YD; 7 ad., 1 juv. (HNHM chilo-7666), ravine, steppe, under stones, N42 $17^{\prime} 53.8^{\prime \prime}$, E6 $67^{\circ} 46^{\prime} 21.0^{\prime \prime}, 314 \mathrm{~m}$ a.s.1., 28.IV.2017, col. YD; 6 ad. (ASU No. 188), 28.IV.2017, col. YD; 3 ad. (HNHM chilo7669), ravine, steppe, under stones, N42 $18^{\prime} 19.5^{\prime \prime}$, E67 $46^{\prime} 28.1^{\prime \prime}$, 292 m a.s.1., 2.V.2017, col. YD; 1 ad. (ASU No. 200), ravine, steppe, under stones, $\mathrm{N} 42^{\circ} 18^{\prime} 19.5^{\prime \prime}, \mathrm{E} 67^{\circ} 46^{\prime} 28.1^{\prime \prime}, 292 \mathrm{~m}$ a.s.1., 2.V.2017, col. YD; 3 ad., 3 juv. (HNHM chilo-7664), near TabakBulak Village, steppe, under bricks and planks, N42 $20^{\prime} 40.4^{\prime \prime}$, E67'38'24.3", 228 m a.s.1., 29.IV.2017, col. YD; 1 ad. (ASU No. 199), Syrdarya-Turkistan State Regional Park, $12 \mathrm{~km} \mathrm{~S}$ of Koksaray Village, Syrdarya River floodplain, N42 $32^{\prime} 46.1^{\prime \prime}$, E68 $10^{\prime} 42.7^{\prime \prime}$, $191 \mathrm{~m}$ a.s.1., 28.V.2017, col. YD; 1 ad. (HNHM chilo-7672), Beltau Mts, steppe, under stones, N41 ${ }^{\circ} 50^{\prime} 28.5^{\prime \prime}$, E6 $68^{\circ} 32^{\prime} 30.3^{\prime \prime}$, 392 m a.s.l., 12-13.V.2017, col. YD; 3 ad. (ZMMU Rc 6230), near Arys Town, steppe, Artemisia, in soil cracks, 24.IV.-5.V.1988, col. DL; 2 ad., 2 sad., (ZMMU Rc 7784), Karatau Mt Range, 9.V.2010, 
col. TT; 2 ad. (ZMMU Rc 7785), near Tabakbulak Village, 2.V.2010, col. TT; Kyzylorda Region, Aral District, Barsa-Kelmes (former island): 2 ad. (ZIN), flat bottom land, 3.V.1983, col. DP; 1 ad. (ZIN), 1.V.1983, col. TVP; 1 ad. (ZIN), 9.V.1983, col. TVP; 1 ad (ZIN), V.1983, col. TVP; 1 ad. (ZIN), 8.V.1983, TVP; 1 ex. (ZIN), 1.V.1983, TVP; 1 ex. (ZIN), 8.V.1983, col. TVP; West Kazakhstan Region, Zhanybek District, Zhanybek Research Station: 1 ad. (ZMMU Rc 6035), 28.III.1976; 2 ad. (ZMMU Rc 6038), 25.I. 1982, col. KM; 1 ad. (ZMMU Rc 6073), Artemisia, 12.IX.1982, col. KM; 4 ad. (ZMMU Rc 6186), Artemisia, 17.VIII-7.IX.1984, col. KM; 1 ad. (ZMMU Rc 6247), Zhanybek, Artemisia, 11.VI.1982, col. KM; 1 ad. (ZMMU Rc 6248), 30.VIII.1982, col. KM; 1 ad. (ZMMU Rc 6249), 26.VIII.1982, col. KM; 1 ad. (ZMMU Rc 6250), 23.VI.1982, col. KM; 1 ad. (ZMMU Rc 6251), 4.IX.1982, col. KM; 1 ad. (ZMMU Rc 6252), 10.IX.1982, col. KM; 1 ad. (ZMMU Rc 6253), 12.VI.1982, col. KM; 1 ad. (ZMMU Rc 6254), 1.VI.1982, col. KM; 1 sad. (ZMMU Rc 6975), flat solonets [aridisol], 7.V.1965, col. TP; 1 juv. (ZMMU Rc 7797), soil samples, 12.IX.1982, col. KM.

DESCRIPTION. Body size up to $80 \mathrm{~mm}$, colour yellow. Tooth-plates of forcipular coxosternite each with 4-5 teeth (Fig. 34). Ts 1 of leg 1 with two spurs (Fig. 37). Coxopleural process of ultimate leg-bearing segment with 5-11 spines, also with 1-2 spines at posterior corner of coxopleura (Fig. 36). Ultimate $P$ with numerous (up to 24) ventral spines, corner spine with 2-6 spinules (Fig. 35).

DISTRIBUTION. This species occurs in Southern Europe (Italy, Greece), North Africa (Morocco, Algeria, Tunisia, Libya, and Egypt), western Asia (Armenia, Azerbaijan, Turkey, Israel, Jordan, Lebanon, Syria, Iran, Saudi Arabia, Yemen) and Central Asia (Afghanistan, Turkmenistan, Uzbekistan, Tajikistan and Kazakhstan) [Lewis, 2001, 2010; Akkari et al., 2008; Simiakis, Mylonas, 2008]. In Kazakhstan: West Kazakhstan [Vsevolodova-Perel, 2009], Mangystau [Kessler, 1874; Sseliwanoff, 1884; Zalesskaja, Schileyko, 1991, 1992; Dyachkov, 2019], Kyzylorda and Turkistan regions.

REMARKS. The specimens studied correspond well to the descriptions of Attems [1930], Würmli [1980], Lewis [1986], and Zalesskaja \& Schileyko [1991]. This species is new to the fauna of the Kyzylorda and Turkistan regions.

\section{Conclusions}

The order Scutigeromorpha is represented in Kazakhstan by three members of the family Scutigeridae: Allothereua kirgisorum, Thereuonema turkestana and Scutigera coleoptrata, the latter species being strictly synanthropic. The taxonomic status of $A$. kirgisorum requires revision. The scolopendromorph fauna of Kazakhstan includes Cryptops doriae, C. hortensis (both Cryptopidae) and Scolopendra canidens (Scolopendridae).

KEY TO THE SCUTIGEROMORPH AND SCOLOPENDROMORPH SPECIES FROM KAZAKHSTAN

1. Body with 15 pairs of legs .................................... 2

- Body with 21 pairs of legs .

4

2. Distal end of tarsus 1 of legs 6-14 each with a pair of spine bristles (Fig. 5) .................. Scutigera coleoptrata

- Tarsi of all legs lacking a pair of spine bristles (Fig. 6) . 3

3. Tergal spiculae short and stout (Fig. 8)

- Tergal spiculae long, needle-shaped (Fig. 4) Allothereua kirgisorum Thereuonema turkestana
4. Ocelli present ............................... Scolopendra canidens

- Ocelli absent .............................................................. 5

5. Femur of ultimate leg lacking saw teeth, tibia with 5-8, tarsus 1 with 2-4 teeth (Fig. 33) ..... Cryptops hortensis - Femur of ultimate leg with a single saw tooth, tibia with 68, tarsus 1 with 3-4 teeth (Fig. 28) ..... Cryptops doriae

Acknowledgments. I am most grateful to S.I. Golovatch (Moscow) who kindly checked the English of an advanced draft. I wish to thank A.A. Schileyko (ZMMU, Moscow) for the identification of Cryptops doriae, correcting an early draft of the manuscript, and the advice on ZMMU material. I am also highly obliged to all collectors who loaned material for study: A.A. Fomichev (Barnaul, Russia), I.I. Temreshev (Almaty, Kazakhstan), G.Sh. Farzalieva (Perm, Russia), V.V. Rudoi (Shemonaikha, Kazakhstan) and several ASU students. The author is most thankful to R.V. Yakovlev (Barnaul, Russia), S.V. Kornev (Orenburg, Russia), D.F. Shovkoon (Samara, Russia) and again I.I. Temreshev who organized my field trips to Kazakhstan. My sincerest thanks go to the administrations and research departments of the Sayram-Ugam National Park, Syrdarya-Turkistan State Regional Park (both Shymkent, Kazakhstan), Karatau Nature Reserve (Kentau, Kazakhstan) and Ustyurt State Nature Reserve (Zhanaozen, Kazakhstan) for their assistance and contributions to my field work. I wish to thank J.A. Dunlop and F. Tillack (both Berlin) for the provision of material from ZMB and for their logistic help. I also wish to thank V.A. Krivokhatsky (Saint Petersburg, Russia) for the provision of material from the ZIN collection. Alessandro Minelli (Padua, Italy) kindly reviewed an advanced draft.

\section{References}

Akkari N., Stoev P., Lewis J.G.E. 2008. The scolopendromorph centipedes (Chilopoda, Scolopendromorpha) of Tunisia: taxonomy, distribution and habitats // ZooKeys. Vol.3. P.77-102. doi: $10.3897 /$ zookeys.3.51

Attems C. 1904. Central- und hoch-asiatische Myriopoden // Zoologische Jahrbücher, Abtheilung für Systematik, Geographie und Biologie der Thiere. Bd.20. S.113-130.

Attems C. 1930. Myriopoda. 2. Scolopendromorpha // Das Tierreich. Lfg.54. $308 \mathrm{~S}$

Barber A.D. 2009. Centipedes. Keys and Notes for Identification of the Species. (Synopses of the British Fauna (New Series), 58). Shrewsbury: Field Studies Council. 127 pp.

Bonato L., Edgecombe G.D., Lewis J.G., Minelli A., Pereira L.A., Shelley R.M., Zapparoli M. 2010. A common terminology for the external anatomy of centipedes (Chilopoda) // ZooKeys. Vol.69. P.17-51. doi: 10.3897/zookeys.69.737

Bonato L., Chagas Junior A., Edgecombe G.D., Lewis J.G.E., Minelli A., Pereira L.A., Shelley R.M., Stoev P., Zapparoli M. 2016. ChiloBase 2.0 - A World Catalogue of Centipedes (Chilopoda). Available at http://chilobase.biologia.unipd.it.

Bragina T.M., Dyachkov Yu.V., Farzalieva G.Sh. 2020. New data on the centipede fauna (Myriapoda: Chilopoda) of Kostanay region, Kazakhstan // Far Eastern Entomologist. No.406. P.2732. https://doi.org/10.25221/fee. 406.4

Demir M. 1948. Türkiye Scutigerid'leri hakkinda. Über türkische Scutigeriden // Revue de la Faculté des Sciences de 1'Université d'Istanbul, Série. B: Sciences naturelles. T.13. F.4. P.269-279.

Dobroruka L.J. 1979. Allothereua wilsonae sp.n., a new centipede from Nepal (Chilopoda: Scutigeromorpha: Thereuoneminae) // Vestnik Ceskoslovenské Spolecnosti Zoologické. Svazek 18. Č́st 2. S.101-103.

Donovan E. 1810. The Natural History of British Insects. Vol.14. London. P.23-24.

Dunlop J.A., Friederichs A., Langermann J. 2017. A catalogue of the scutigeromorph centipedes in the Museum für Naturkunde, 
Berlin // Zoosystematics and Evolution. Vol.93. No.2. P.281295.

Dyachkov Yu. V. 2019. The centipede (Chilopoda) fauna of Kazakhstan // 18th International Congress of Myriapodology. Program and Abstracts. Budapest: Hungarian Natural History Museum \& Hungarian Biological Society. P.92.

Dyachkov Yu.V., Farzalieva G.Sh., Fomichev A.A. 2016. New data on the centipede (Chilopoda) fauna of East Kazakhstan region // Biological Bulletin of Bogdan Chmelnitskiy Melitopol State University. Vol.6. No.3. P.438-442. doi: 10.15421/ 2016115

Edgecombe G.D., Giribet G. 2009. Phylogenetics of scutigeromorph centipedes (Myriapoda: Chilopoda) with implications for species delimitation and historical biogeography of the Australian and New Caledonian faunas // Cladistics. Vol.25. No.4. P.406-427. DOI: $10.1111 / \mathrm{j} .1096-0031.2009 .00253 . \mathrm{x}$

Edgecombe G.D. 2011. [Order Scutigeromorpha] // A. Minelli (ed.). Treatise on Zoology - Anatomy, Taxonomy, Biology. The Myriapoda. Vol.1. Leiden-Boston: Brill. P.363-370.

Farzalieva G.Sh. 2008. [The fauna and chorology of Myriapoda from the Urals and Cisuralia]. Thesis of Candidate (Ph.D.) of Biological Sciences Degree. Perm State University. 189 pp. [In Russian]

Faúndez E.I. 2011. On the presence of Scutigera coleoptrata (Linnaeus, 1758) (Chilopoda: Scutigeromorpha: Scutigeridae) in the Metropolitan Region, Chile // Boletín de la Sociedad Entomológica Aragonesa. Vol.49. P.336.

Giribet G., Edgecombe G.D. 2013. Stable phylogenetic patterns in scutigeromorph centipedes (Myriapoda: Chilopoda: Scutigeromorpha): dating the diversification of an ancient lineage of terrestrial arthropods // Invertebrate Systematics. Vol.27. P.485501.

Kessler K.F. 1874. [On Russian Scolopendridae and Geophilidae] // Trudy Russkogo Entomologicheskogo obshchestva. St. Petersburg. Vol.8. P.28-45 [in Russian].

Kraepelin K. 1903. Revision der Scolopendriden // Mitteilungen aus dem Naturhistorischen Museum in Hamburg. Vol.20. P.1276.

Lewis J.G.E. 1981. The Biology of Centipedes. Cambridge - New York: Cambridge University Press. 476 pp.

Lewis J.G.E. 1986. Centipedes of Saudi Arabia // Fauna of Saudi Arabia. Vol.8. P. 20-30.

Lewis J.G.E. 1999. On the genus Cryptops Leach in Nepal with redescriptions of Cryptops australis Newport and C. doriae Pocock (Chilopoda, Scolopendromorpha, Cryptopidae) // Seckenbergiana biologica. Bd.79. S.19-38.

Lewis J.G.E. 2001. Scolopendromorph centipedes (Chilopoda: Scolopendromorpha) collected in northern Iraq by Dr Hywel Roberts with a review of previous records // Arthropoda Selecta. Vol.10. No.2. P.151-154.

Lewis J.G.E. 2007. On Cryptops doriae Pocock from the wet tropical biome of the Eden Project, Cornwall (Chilopoda, Scolopendromorpha, Cryptopidae) // Bulletin of the British Myriapod \& Isopod Group. Vol.22. P.12-16.

Lewis J.G.E. 2010. A key and annotated list of the Scolopendra species of the Old World with a reappraisal of Arthrorhabdus (Chilopoda: Scolopendromorpha: Scolopendridae) // International Journal of Myriapodology. Vol.3. P.83-122. doi: 10.1163/ $187525410 X 12578602960380$

Lewis J.G.E. 2011. A review of the species in the genus Cryptops Leach, 1815 from the Old World related to Cryptops (Cryptops) hortensis (Donovan, 1810) (Chilopoda, Scolopendromorpha) // International Journal of Myriapodology. Vol.4. P.1150. doi: $10.3897 / \mathrm{ijm} \cdot 4.1116$

Lewis J.G.E. 2013. A review of the species in the genus Cryptops Leach, 1815 from the Old World and the Australasian region related to Cryptops (Cryptops) doriae Pocock, 1891 (Chilopoda: Scolopendromorpha: Cryptopidae) // Zootaxa. Vol.3683. No.1. P.1-34. doi: 10.11646/zootaxa.3683.1.1

Lignau N.G. 1929a. Zur Kenntnis der zentralasiatischen Myriopoden // Zoologischer Anzeiger. Bd.85. H.5/8. S.159-175.

Lignau N.G. 1929b. Neue Myriopoden aus Zentralasien // Zoologischer Anzeiger. Bd.85. H.9/10. S.205-217.
Loksa I. 1971. Die von K. Lindberg in Afghanistan gesammelten Chilopoden // Senckenbergiana biologica. Bd.52. H.1/6. S.103112.

Nefediev P.S., Tuf I.H., Dyachkov Yu.V., Efimov D.A. $2016 \mathrm{a}$. First record of Scutigera coleoptrata (Linnaeus, 1758) in the south of Western Siberia, Russia (Chilopoda: Scutigeromorpha: Scutigeridae) // Biological Bulletin of Bogdan Chmelnitskiy Melitopol State Pedagogical University. Vol.1. No.6. P. 428-432, doi: $10.15421 / 201626$

Nefediev P.S., Tuf I.H., Dyachkov Yu.V. 2016b. First record of Cryptops (Cryptops) hortensis (Donovan, 1881) in Southwestern Siberia, Russia (Chilopoda: Scolopendromorpha: Cryptopidae) // Biological Bulletin of Bogdan Chmelnitskiy Melitopol State Pedagogical University. Vol.2. No.6. P.107-109. doi: 10. $15421 / 201642$

Ostrovsky A.M. 2016. The first records of Scutigera coleoptrata (Linnaeus, 1758) (Chilopoda: Scutigeromorpha: Scutigeridae) from Belarus // Arthropoda Selecta. Vol.25. No.4. P.355-356.

Ostrovsky A.M. 2018. The first record of Cryptops hortensis (Donovan, 1810) (Chilopoda: Scolopendromorpha: Cryptopidae) in Belarus // Arthropoda Selecta. Vol.27. No.1. P.31-32. doi: 10.15298/arthsel. 27.1.03

Schileyko A.A. 2007. The scolopendromorph centipedes (Chilopoda) of Vietnam, with contributions to the faunas of Cambodia and Laos. Part 3 // Arthropoda Selecta. Vol.16. No.2. P.71-95.

Schileyko A.A., Stoev P. 2016. Schileyko. Scolopendromorpha of New Guinea and adjacent islands (Myriapoda, Chilopoda). Zootaxa. Vol.4147. No.3. P.247-280.

Shnitnikov V.N. 1925. [Dzhetysu (Semirechie). A natural and historical description of the region]. Tashkent: Uzbekskkoye gosudarstvennoye izdatelstvo. 234 pp. [In Russian]

Shnitnikov V.N. 1928. [Reptiles of the Semirechye] // Trudy obschestva izucheniya Kazakhstana. Kzyl-Orda. T.8. No.3. 85 pp. [In Russian]

Shnitnikov V.N. 1934. [The world of animals of Kazakhstan. Pt. I. Southern Kazakhstan]. Alma-Ata: Kazakhstkoye kraevoye izdatelstvo. 200 pp. [In Russian]

Shnitnikov V.N. 1958. [From the memories of a naturalist]. Moscow: Gosudarstvennoe izdatelstvo geographicheskoy literatury. 326 pp. [In Russian]

Sseliwanoff A.V. 1884. [Materials towards the study of Russian myriapods] // Trudy Russkago Entomologicheskago obshchestva. St. Petersburg. T.18. P.69-121 [in Russian].

Simiakis S., Mylonas M. 2008. The Scolopendra species (Chilopoda: Scolopendromorpha: Scolopendridae) of Greece (E-Mediterranean): a theoretical approach on the effect of geography and paleography on their distribution // Zootaxa. Vol.1792. P.39-53. doi: 10.5281/zenodo. 182561

Stoev P. 2002. The scutigeromorphs (Chilopoda: Scutigeromorpha) in the collection of the National Museum of Natural History, Sofia // Historia naturalis bulgarica. Vol.15. P.79-85.

Stoev P., Geoffroy J.-J. 2004. An annotated catalogue of the scutigeromorph centipedes in the collection of the Muséum National d'Histoire Naturelle, Paris (France) (Chilopoda: Scutigeromorpha) // Zootaxa. Vol.635. P.1-12.

Tran B.T., Le S.X., Nguyen A.D. 2013. An annotated checklist of centipedes (Chilopoda) of Vietnam // Zootaxa. Vol.3722. No.2. P.219-244. doi: 10.11646/zootaxa.3722.2.6

Verhoeff C.W. 1905. Über Scutigeriden. 5. Aufsatz // Zoologischer Anzeiger. Bd.29. H.2/3. P.73-119.

Volkova Yu.S. 2014. [The fauna of predatory myriapods (Chilopoda) of the Ulyanovsk Area] // Priroda Simbirskogo Povolzh'ya. Ulyanovsk. Vol.15. P.95-103 [in Russian].

Vsevolodova-Perel T.S. 2009. [Composition of soil populations of clayey semidesert. Ecological and faunistic characterization of soil-dwelling invertebrates] // A.A. Tishkov (ed.). Zhivotnye glinistoi polupustyni Zavolzh'ya (konspekty faun i ekologicheskie kharakteristiki). Moscow: KMK Scientific Press. P.137-138 [in Russian].

Würmli M. 1975. Revision der Hundertfüsser-Gattung Thereuonema (Chilopoda: Scutigeridae) // Entomologica Germanica. Vol.2. No.2. P.189-196. 
Würmli M. 1980. Statistische Unterschungen zur Systematik und postembryonalen Entwicklung des Scolopendra canidens Gruppe (Chilopoda: Scolopendromorpha) // Sitzungsberichte der Österreichischen Akademie der Wissenschaften. Bd.189. S.315-353.

Zalesskaja N.T, Schileyko A.A. 1991. [The scolopendromorph centipedes (Chilopoda, Scolopendromorpha)]. Moscow: Nauka Publ. 102 pp. [In Russian]

Zalesskaja N.T., Schileyko A.A. 1992. The distribution of Scolopendromorpha in the USSR (Chilopoda) // Berichte des natur- wissenschaftlichen-medizinischen Vereins Innsbruck. Suppl.10. P.367-372.

Zuev R.V. 2016. Centipedes (Chilopoda) from the Stavropol Territory, northern Caucasus, Russia // Arthropoda Selecta. Vol.25. No.1. P.23-38.

Zuev R.V., Evsyukov A.P. 2016. Centipedes (Chilopoda) from the Rostov-on-Don Region, southern Russia // Russian Entomological Journal. Vol.25. No.4. P.417-426.

Responsible editor S.I. Golovatch 